\title{
Cataract Surgery Practices in the Republic of Korea: A Survey of the Korean Society of Cataract and Refractive Surgery 2018
}

\author{
Jin Kwon Chung ${ }^{1}$, Hyung Keun Lee ${ }^{2}$, Mee Kum Kim³ ${ }^{3}$ Hong Kyun $\mathrm{Kim}^{4}$, Sun Woong Kim ${ }^{5}$ Eun Chul Kim ${ }^{6}$, \\ Hyun Seung $\mathrm{Kim}^{7}$ \\ ${ }^{I}$ Department of Ophthalmology, Soonchunhyang University College of Medicine, Seoul, Korea \\ ${ }^{2}$ Department of Ophthalmology, Yeonsei University College of Medicine, Seoul, Korea \\ ${ }^{3}$ Department of Ophthalmology, Seoul National University College of Medicine, Seoul, Korea \\ ${ }^{4}$ Department of Ophthalmology, Kyungpook National University College of Medicine, Daegu, Korea \\ ${ }^{5}$ Department of Ophthalmology, Yeonsei University Wonju College of Medicine, Wonju, Korea \\ ${ }^{6}$ Department of Ophthalmology, Bucheon St. Mary's Hospital, The Catholic University of Korea College of Medicine, Bucheon, Korea \\ ${ }^{7}$ Department of Ophthalmology, Seoul St. Mary's Hospital, The Catholic University of Korea College of Medicine, Seoul, Korea
}

Purpose: To describe current cataract surgery practice patterns and changing trends among Korean ophthalmologists.

Methods: A survey of members of the Korean Society of Cataract and Refractive Surgery was performed in July 2018. One hundred and two (12.7\%) of 801 questionnaires were returned for analysis. The data were analyzed using descriptive statistics and compared with previous surveys.

Results: Most of the respondents (75\%) had been in practice for 6 or more years and performed an average of 31 cataract surgeries per month. The preferred method for cataract surgery was phacoemulsification (95\%); $5 \%$ used a femtosecond laser. The use of topical anesthesia markedly increased from $69 \%$ (2012) to $80 \%$ (2018). The use of optical biometry exceeded that of ultrasound A-scan biometry. A multifocal intraocular lens was used by $76 \%$ of the respondents compared with $44 \%$ of the respondents in 2012 . Topical nonsteroidal anti-inflammatory drugs were used by $70 \%$ of the respondents postoperatively. Most $(59 \%)$ of these anti-inflammatory drugs were prescribed for 4 weeks.

Conclusions: This survey provided a comprehensive update of the present cataract surgery practices in the Republic of Korea. The results emphasized the increasing use of premium intraocular lenses, optical biometry, and topical anesthesia.

Key Words: Biometry, Cataract, Intraocular lenses, Phacoemulsification, Surveys and questionnaires

Received: June 13, 2019 Final revision: July 9, 2019 Accepted: July 16, 2019

Corresponding Authors: Jin Kwon Chung, MD, PhD. Department of Ophthalmology, Soonchunhyang University Seoul Hospital, 59 Daesagwan-ro, Yongsan-gu, Seoul 04401, Korea. Tel: 82-2-709-9354, Fax: 82-2-710-3196, E-mail: schcornea@schmc.ac.kr

Hyun Seung Kim, MD, PhD. Department of Ophthalmology, Seoul St. Mary's Hospital, The Catholic University of Korea College of Medicine, 222 Banpo-daero, Seocho-gu, Seoul 06591, Korea. Tel: 82-2-2258-1188, Fax: 82-2-599-7405, E-mail: sara514@catholic.ac.kr 
Age-related cataracts are the leading cause of visual impairment, and cataract extraction is the most commonly performed eye operation in the world [1]. Preferred surgical techniques, phacoemulsification equipment, and intraocular lenses (IOLs) have changed over the past years with associated improvements in outcomes and safety [2]. Many surveys have been reported regarding current practice and trends in cataract surgery in the USA, Canada, Japan, and New Zealand [3-5].

Current practices and trends in cataract surgery have been assessed by the Korean Society of Cataract and Refractive Surgery (KSCRS) since 1995, with the latest results based on the 2012 survey of cataract surgical techniques, IOL preferences, and miscellaneous contemporary issues [6-8]. An understanding of the current practices for cataract surgery is important because of the rapidly changing nature of cataract surgery. These practices are driven by cost, efficiency, and improved patient-centered care $[9,10]$.

In this study, we analyzed the survey results of KSCRS members in 2018 regarding the types of cataract surgery performed, the types of implanted IOLs, surgical procedures, postoperative management, and the frequency of complications to create a comprehensive picture of current cataract surgical practice in the Republic of Korea.

\section{Materials and Methods}

In July 2018, 32 multiple choice or open-ended questionnaires were sent via e-mail to 801 members of the KSCRS. The questionnaire used in this study was based on a previous KSCRS survey with some modifications to identify the latest and changing surgical trends, such as newly developed types of multifocal IOLs and biometry equipment or femtosecond laser-assisted cataract surgery (FLACS) [7]. The questionnaire consisted of three major categories: surgeon demographics, cataract surgery-general, cataract surgery-skill, and related complications. Return questionnaires were not marked or labeled to maintain the confidentiality of the respondent, and no financial reward was offered. One hundred and two (12.7\%) members completed the questionnaire. IBM SPSS Statistics ver. 20.0 (IBM Corp., Armonk, NY, USA) was used for statistical analysis.

\section{Results}

\section{Surgeon demographics}

Most respondents were 30 to 49 years of age (88\%), male (78\%), and had more than 6 years of surgical experience (75\%). Most respondents also worked for university hospitals (48\%), followed by private clinics (31.4\%), and eye hospitals $(15 \%)$. The respondent demographics are shown in Table 1.

Table 1. Characteristics of ophthalmologists who participated in the cataract surgery survey

\begin{tabular}{lc}
\hline Characteristics & Value \\
\hline Age (yr) & $31(30.4)$ \\
$30-39$ & $59(57.8)$ \\
$40-49$ & $11(10.8)$ \\
$50-59$ & $0(0)$ \\
$60-69$ & $1(1.0)$ \\
$70-79$ & \\
Workplace & $32(31.4)$ \\
Private clinic & $2(2.0)$ \\
Public health clinic or & \\
military hospital & $1(1.0)$ \\
National hospital & $15(14.7)$ \\
Eye hospital & $3(2.9)$ \\
General hospital & $49(48.0)$ \\
University hospital & \\
Sex & \\
Male : female & \\
Carrier of surgical practice (yr) & $(78.4): 22(21.6)$ \\
$\leq 5$ & \\
$6-10$ & $26(25.5)$ \\
$11-20$ & $27(26.5)$ \\
No. of teammate doctors & $38(37.3)$ \\
$2-4$ & $11(10.8)$ \\
$5-9$ & \\
\hline & \\
& \\
&
\end{tabular}

Values are presented as number (\%). 


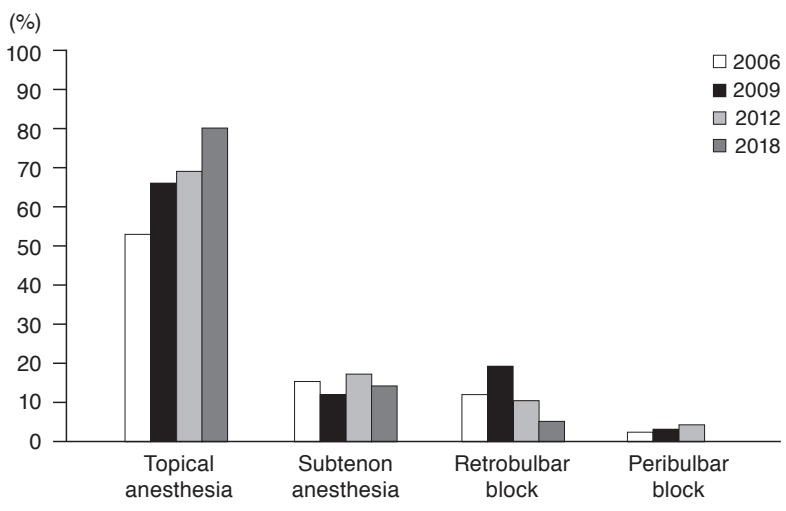

Fig. 1. Changing trends of preferred anesthesia techniques used for cataract surgery.

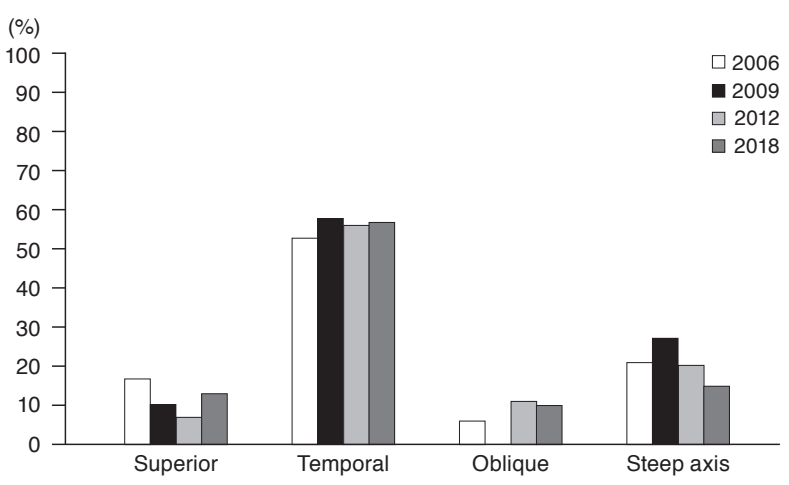

Fig. 2. Changing trends of the preferred incision meridian.

\section{Cataract surgery-general}

The average monthly volume of cataract surgeries performed by KSCRS members was 31 cases. Seventy-two percent performed 1 to 5 post refractive surgeries per month, $5 \%$ performed 6 to 10 surgeries, $2 \%$ performed $>10$ surgeries, and $21 \%$ did not perform post refractive surgery. These surgeons preferred phacoemulsification (95\%) and $5 \%$ used a femtosecond laser. None of the members performed planned extracapsular cataract extraction. Surgeons were assisted during the procedures by residents $(41 \%)$, nurses $(16 \%)$, and nursing assistants $(15 \%)$. The surgical instruments were managed and sterilized by nurses $(83 \%)$ and nursing assistants (15\%). Ninety-four percent of the respondents selected the IOL power, and $4 \%$ of the respondents had the residents perform this task.

\section{Biometry for axial length measurements}

Optical biometry was used by $92 \%$ of the respondents for IOL calculations, and ultrasound biometry was used by
$73 \%$ of the respondents. Seventy-eight percent of respondents used IOLmaster (Carl Zeiss Meditech, Jena, Germany), 73\% used ultrasound A-scan biometry, 14\% used Lenstar (Haag-Streit, Koeniz, Switzerland), and 8\% used ALscan (Nidek, Gamamori, Japan). Thirty-four percent of the respondents used single equipment, $23 \%$ used an IOLmaster, and $8 \%$ used an ultrasound A-scan. Sixty-six percent used more than two instruments; the most common combination was ultrasound A-scan biometry and the IOLmaster $(44 \%)$.

\section{Anesthesia}

Topical anesthesia was used by $80 \%$ of the respondents (from $69 \%$ in 2012). Subtenon anesthesia was used by $14 \%$ of the respondents (from 17\% in 2012), and retrobulbar block was used by $5 \%$ (from $10 \%$ in 2012) (Fig. 1) [7].

\section{Cataract surgery-technique}

Temporal clear corneal incisions were used by $50 \%$ of the respondents, followed by a steep axis incision (15\%). The temporal site was the preferred incision meridian (57\%), with little variation since 2006 (Fig. 2) [7]. A cataract incision size of $2.8 \mathrm{~mm}$ was used by $64 \%$ of the respondents (from 61\% in 2012), and a size of $2.2 \mathrm{~mm}$ was used by $31 \%$ (from $24 \%$ in 2012) [7]. Among ophthalmic viscoelastic devices, HEALON (Johnson \& Johnson, New Brunswick, NJ, USA) was used by $65 \%$ of the respondents; HEALON GV (Johnson \& Johnson) was used by 10\%; Viscoat (Alcon Laboratories, Fort Worth, TX, USA) was used by 7\%; and HEALON 5 (Johnson \& Johnson) was used by $3 \%$. Forceps were used by $68 \%$ of the respondents for continuous curvilinear capsulorhexis, a bent needle was used by $24 \%$, and a femtosecond laser was used by $2 \%$. Capsular polishing was performed by $95 \%$ of the respondents during surgery; $5 \%$ of the respondents did not use this technique. Forty-eight percent of the respondents used both anterior and posterior capsules, while $44 \%$ and $3 \%$ of the respondents used only an anterior capsule or posterior capsule, respectively.

\section{IOL use}

In 2001, an acrylic IOL was the preferred optic material, exceeding half of the total number of IOLs used (Fig. 3) [6]. 


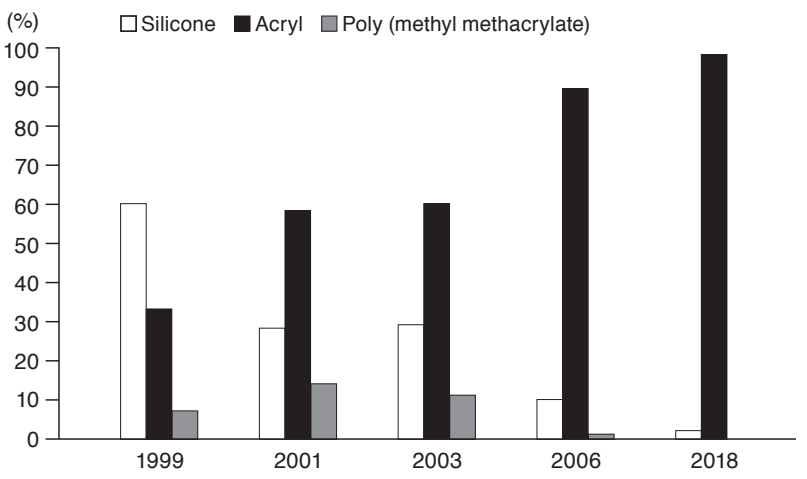

Fig. 3. Changing trends of the preferred intraocular lens material.
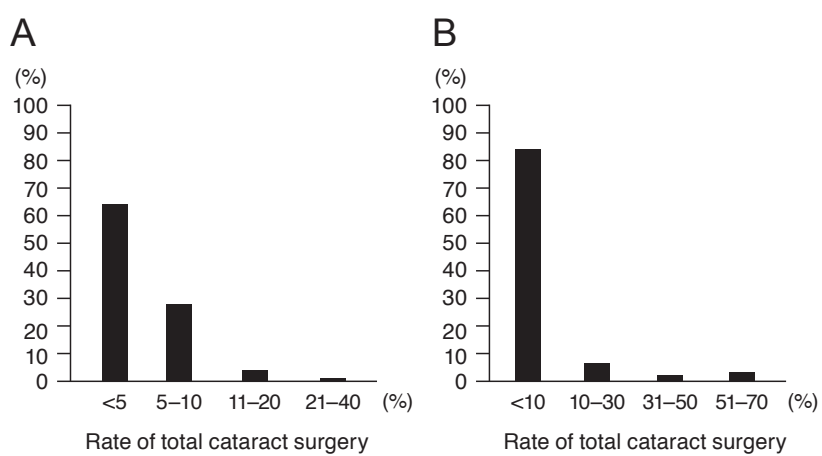

Fig. 4. Percentages of (A) toric intraocual lens and (B) multifocal intraocual lens implanted after phacoemulsification among the total number of cataract surgeries.

Table 2. The preferred multifocal intraocular lens of respondents

\begin{tabular}{lc}
\hline Name of intraocular lens & Value \\
\hline TECNIS Symfony & $21(30)$ \\
AcrySof IQ ReSTOR & $20(28)$ \\
TECNIS Multifocal & $18(25)$ \\
AT LISA tri & $13(18)$ \\
AcrySof PanOptix & $12(17)$ \\
Lentis Mplus & $7(10)$ \\
FineVision POD F & $6(9)$ \\
Precizon Presbyopic & $2(3)$ \\
Bi-Flex M & $1(1)$ \\
Reviol Tri-ED & $1(1)$ \\
\hline
\end{tabular}

Values are presented as number (\%).

In 2018 , acrylic was preferred by $98 \%$ of the respondents and silicone by $2 \%$; poly (methyl methacrylate) was not used by any respondent $(0 \%)$. Among the total number of cataract surgeries, $64 \%$ of the respondents used a toric IOL less than $5 \%$, and $84 \%$ used multifocal IOLs less than $10 \%$ (Fig. 4). Twenty-four percent of the respondents did not use multifocal IOLs; and $66 \%$ of the respondents treated less than 10 cases per month, while $8 \%$ treated $10-50$ cases per month. Eighty-seven percent of the respondents also used multifocal IOLs for patients who were treated with corneal refractive surgery, and it constituted less than $30 \%$ of the total cataract surgery cases.

Eighty-two percent of the respondents using multifocal IOLs had been using bifocal IOLs, $48 \%$ had been using trifocal IOLs, and $30 \%$ had been using both types of IOLs. The types of preferred multifocal IOLs are shown in Table 2.

\section{Postoperative management}

Forty-seven percent of the respondents did not use oral antibiotics postoperatively, and $31 \%$ used oral antibiotics postoperatively for less than 3 days. Forty-one percent of the respondents (from $77 \%$ in 2012) prescribed antibiotics for 3 to 7 days. Topical nonsteroidal anti-inflammatory drugs (NSAIDs) were used together with topical steroids by $60 \%$ of the respondents, $10 \%$ used topical NSAIDs alone, and 30\% did not use topical NSAIDs. More than half $(53 \%)$ of the respondents used topical antibiotics and steroids for 1 month, and $26 \%$ of the respondents continued to use topical antibiotics and steroids longer than 1 month. In a similar manner, $52 \%$ of the respondents used topical antibiotics and NSAIDs for 1 month postoperatively. Twenty percent of the respondents finished follow-up visits at 1 month postoperatively, 30\% finished visits at 3 months, $15 \%$ finished visits at 6 months, and $26 \%$ continued to visit every year. Most respondents (72\%) prescribed reading glasses at 1 month postoperatively.

\section{Complications}

Ninety-four percent of the respondents (from $80 \%$ in 2012) observed posterior capsular rupture during surgery in less than $5 \%$ of cases, and $71 \%$ observed posterior capsular rupture during surgery in less than $1 \%$ of cases. More than half (54\%) of the respondents did not observe patients with postoperative endophthalmitis, $23 \%$ observed postoperative endophthalmitis once, and $23 \%$ observed postoperative endophthalmitis in less than five cases. Fifty-eight 


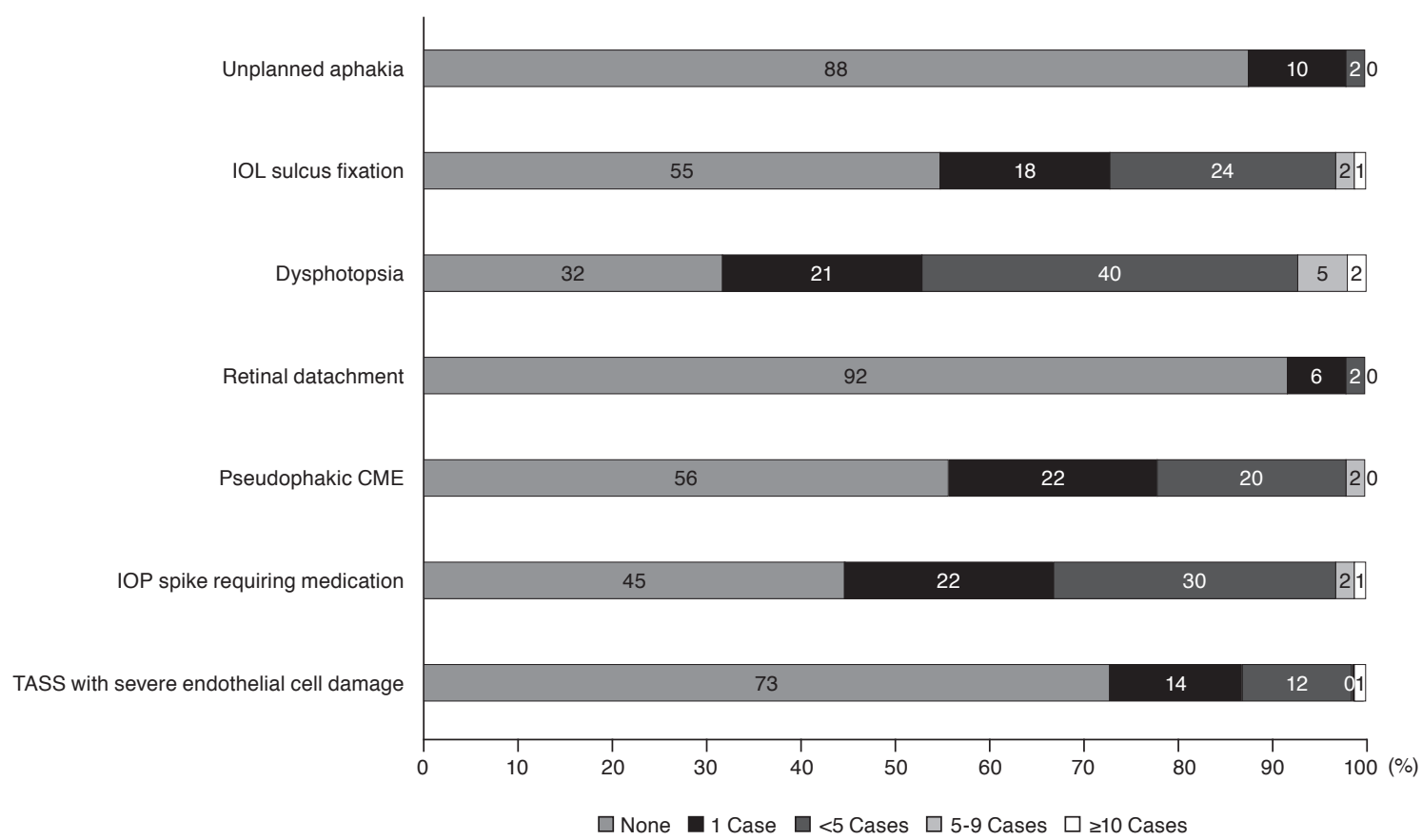

Fig. 5. The complication rate of toxic anterior segment syndrome (TASS) with severe endothelial cell damage, intraocular pressure (IOP) spikes requiring medication, clinically significant pseudophakic cystoid macular edema (CME), retinal detachment, intraocular lens (IOL) subluxation/dislocation, dysphotopsia, IOL sulcus fixation, and unplanned aphakia.

percent of those who observed postoperative endophthalmitis estimated that the occurrence was less than $0.1 \%$. More than half (52\%) of the respondents observed severe postoperative uveitis, and $63 \%$ of these respondents estimated that the occurrence was less than $0.1 \%$ of cases.

The occurrences of other complications such as toxic anterior segment syndrome with severe endothelial cell damage, intraocular pressure spikes requiring medication, clinically significant pseudophakic cystoid macular edema, retinal detachment, dysphotopsia, IOL sulcus fixation, and unplanned aphakia are shown in Fig. 5. More than half $(53 \%)$ of the respondents did not observe IOL subluxation or dislocation in uncomplicated cases over the past 10 years, $16 \%$ observed this complication once, $31 \%$ observed this complication in less than five cases, and $1 \%$ observed this complication in 10 cases or more. Among secondary IOL implantation techniques, conventional scleral fixation with suture material was used by $76 \%$ of the respondents; the Hoffmann technique was used by $10 \%$; the iris-fixated IOL technique was used by $10 \%$; and the glued intrascleral haptic fixation was used by $2 \%$.

\section{Discussion}

This survey provides a summary of current practices of KSCRS members performing cataract surgery and describes the changing trends in clinical practice. Most of the respondents $(75 \%)$ had been in practice for 6 or more years and had performed an average of 31 cataract surgeries per month. There was a moderate increase in the number of respondents treating 16 to 50 cases per month from 2012 (55\%) to 2018 (62\%), and over 51 cases from $2012(7 \%)$ to $2018(11 \%)$. There was a decreased number of respondents treating 6-15 cases from $2012(33 \%)$ to $2018(18 \%)$.

This survey involved the latest practice of KSCRS members in cataract surgery; some questions in the survey were modified from their original version used in previous surveys. Some questionnaire items such as FLACS, cataract surgery after refractive surgery, and NSAIDS eye drops were added; and the categories of biometry equipment, incisions, continuous curvilinear capsulorhexis, multifocal IOLs, toric IOLs, and postoperative management were refined [7].

Because no surgeon performed planned extracapsular cataract extraction and the majority (95\%) of respondents 
used phacoemulsification, there was a marked increase in the number of respondents who used topical anesthesia, from $69 \%$ (2012) to $80 \%$ (2018), and a decreased use of retrobulbar block, from $10 \%$ to $5 \%$ [7]. These changes also seemed to affect the preferred smaller size of incisions.

The first report of FLACS was included in the current survey. In the 2012 survey, $32 \%$ of the respondents expressed an interest in FLACS however, was not used in practice. Only 5\% of the respondents in this survey performed FLACS, which showed that FLACS still had a limited role in the Republic of Korea. Possible explanations include the high cost of purchase and operation, as well as the lack of clear advantages of FLACS over traditional phacoemulsification [11-13]. According to the 2018 American Society of Cataract and Refractive Surgery clinical survey, only an average of $8 \%$ of cataract patients were treated using FLACS. USA respondents performed FLACS more often, with $10 \%$ of cataract patients in the USA. Treated with FLACS vs. $6 \%$ of non-USA patients [14]. Another survey from the Canadian Ophthalmological Society (COS) reported that the use of FLACS had increased from $2014(8 \%)$ to 2015 (18.9\%), then started to decline in 2016 (17.4\%) and in 2017 (11.8\%) [15]. However, we cannot predict changes in the use FLACS from the results of this survey alone and additional studies are needed.

There was a marked increase in the number of respondents who were using optical biometry. Optical biometry was used by $92 \%$ of the respondents and ultrasound biometry by $73 \%$ of the respondents. For example, the use of IOLmaster increased from $56 \%$ in 2012 to $78 \%$ in 2018 . As new optical biometry equipment has been introduced with a newer generation of IOL calculation formulas, optical biometry has partially replaced conventional ultrasound A-scan biometry. Optical biometry is convenient to use, reproducible, and has an installed calculation formula. Several favorable results in cases implanted with multifocal or toric IOLs seem to be related to its increasing popularity.

There was a marked increase in the number of respondents who were using multifocal IOLs, from $44 \%$ (2012) to $76 \%$ (2018). This was higher than the COS report of approximately $50 \%$ in 2017; two-thirds was the proportion reported by the American Society of Cataract and Refractive Surgery clinical survey in $2018[14,15]$.

The most current survey included the first use of NSAIDS eye drops. These were used by $70 \%$ of the re- spondents postoperatively, and the majority prescribed eye drops for 4 weeks (59\%). This was similar to the COS report, which showed that $75.9 \%$ of the respondents prescribed NSAIDS and $52.4 \%$ prescribed these drops for 4 weeks [15].

This survey has several limitations. Response bias could have affected the results although there were few open-ended questions. The questionnaire primarily involved multiple choice questions without the option of responses that were not listed. Because the response represented only $12.7 \%$ of the KSCRS members $(20.6 \%$ in 2012), the results may not represent the opinions and practices of the majority of ophthalmologists in the Republic of Korea.

In summary, this study provides a comprehensive update of the present cataract surgery practices of KSCRS members. The results emphasize the growing role of premium IOLs, optical biometry, and the use of topical anesthesia. A follow-up survey concerning the use of FLACS, post refractive cataract surgery, the use of premium IOLs, and changes in postoperative medications is required.

\section{Conflict of Interest}

No potential conflict of interest relevant to this article was reported.

\section{References}

1. Day AC, Gore DM, Bunce C, Evans JR. Laser-assisted cataract surgery versus standard ultrasound phacoemulsification cataract surgery. Cochrane Database Syst Rev 2016;7:CD010735.

2. Reddy KP, Kandulla J, Auffarth GU. Effectiveness and safety of femtosecond laser-assisted lens fragmentation and anterior capsulotomy versus the manual technique in cataract surgery. J Cataract Refract Surg 2013;39:1297-306.

3. Leaming DV. Practice styles and preferences of ASCRS members: 2003 survey. J Cataract Refract Surg 2004;30:892900.

4. Pick ZS, Leaming DV, Elder MJ. The fourth New Zealand cataract and refractive surgery survey: 2007. Clin Exp Ophthalmol 2008;36:604-19.

5. Oshika T, Amano S, Araie M, et al. Current trends in cata- 
ract and refractive surgery in Japan: 1999 survey. Jpn $J$ Ophthalmol 2001;45:383-7.

6. Yoon SC, Jung JW, Sohn HJ, Shyn KH. Cataract and refractive surgery in: a survey of KSCRS members from 1995-2006. Korean J Ophthalmol 2009;23:142-7.

7. Wi JM, Moon HS, Kim KH, Shyn KH. 2012 survey of KSCRS and KOS member: current trends in cataract surgery in Korea. J Korean Ophthalmol Soc 2015;56:1181-7.

8. Shyn KH, Baek NH, Lee JH, et al. 95 survey of Korean Society of Cataract and Refractive Surgery members. $J$ Korean Ophthalmol Soc 1998;39:892-9.

9. Ianchulev T, Litoff D, Ellinger D, et al. Office-based cataract surgery: population health outcomes study of more than 21,000 cases in the United States. Ophthalmology 2016;123:723-8.

10. Chen CL, Lin GA, Bardach NS, et al. Preoperative medical testing in Medicare patients undergoing cataract surgery. $N$ Engl J Med 2015;372:1530-8.
11. Havnaer AG, Greenberg PB, Cockerham GC, et al. Cataract surgery practices in the United States Veterans Health Administration. J Cataract Refract Surg 2017;43:543-51.

12. Popovic M, Campos-Moller X, Schlenker MB, Ahmed II. Efficacy and safety of femtosecond laser-assisted cataract surgery compared with manual cataract surgery: a meta-analysis of 14567 eyes. Ophthalmology 2016;123:2113-26.

13. Chen X, Xiao W, Ye S, et al. Efficacy and safety of femtosecond laser-assisted cataract surgery versus conventional phacoemulsification for cataract: a meta-analysis of randomized controlled trials. Sci Rep 2015;5:13123.

14. Braga-Mele R. ASCRS clinical survey 2018 [Internet]. [place unknown]: Eyeworld News Service; 2018 [cited 2019 Jul 1]. Available from: http://supplements.eyeworld.org/eyeworld-supplements/december-2018-clinical-survey.

15. Ong-Tone L. Practice patterns of Canadian ophthalmological society members in cataract surgery: 2017 survey. Can J Ophthalmol 2018;53:1. 